\title{
Peri-implant Bone Remodeling after Total Hip Replacement Combined with Systemic Alendronate Treatment: A Finite Element Analysis
}

\author{
B. PETER ${ }^{\mathrm{a}}$, N. RAMANIRAKA ${ }^{\mathrm{a}}$, L.R. RAKOTOMANANA ${ }^{\mathrm{b}}$, P.Y. ZAMBELLI ${ }^{\mathrm{c}}$ and D.P. PIOLETTI ${ }^{\mathrm{a}, *}$ \\ ${ }^{\mathrm{a}}$ Center for Orthopedic Research, Swiss Federal Institute of Technology, 1015 Lausanne, Switzerland; \\ bIRMAR, University of Rennes I, Rennes, France; \\ ${ }^{\mathrm{c}}$ Hôpital Orthopédique de la Suisse Romande, 1005 Lausanne, Switzerland
}

(Received 14 July 2003; In final form 5 January 2004)

\begin{abstract}
In order to decrease the peri-implant bone loss during the life-time of the implant, oral use of antiosteoporosis drugs (like bisphosphonates) has been suggested.

In this study, bone remodeling parameters identified from clinical trials of alendronate were used to simulate the effect of those drugs used after total hip arthroplasty on the peri-implant bone density. Results of the simulation show that the oral administrated drugs increase bone density around the implant and decreases, at the same time, the micromovements between the implant and the surrounding bone tissue.

Incorporation of drug effect in numerical studies of bone remodeling is a promising tool especially to predetermine safe bisphosphonate doses that could be used with orthopedic implants.
\end{abstract}

Keywords: Remodeling model; Total hip arthroplasty; Bisphosphonate; Orthopedic implants

\section{INTRODUCTION}

Cementless hip implants are frequently used in young patients. However, failure rates can exceed $30 \%$ after 15 years for patients younger than 50 years old [1]. Clearly, for this situation the long term performance of implants has to be increased.

The main cause of implant failure is loosening following osteolysis caused by a combination of stress shielding [2] and of inflammatory reaction induced by wear particles $[3,4]$. Besides the improvement of the material and wear properties of the implant, biological aspects of peri-implant bone remodeling should be investigated.

The efficiency of bisphosphonate in controlling osteoporosis has been shown by many authors $[5,6]$. Supposing that the same mechanism is driving osteoporosis and peri-implant bone resorption, it was then suggested to use bisphosphonate to control peri-implant osteolysis [7]. This idea is currently tested in clinical studies [8]. However, as two opposing effects are competing, namely osteolysis due to the implant and decreased bone resorption due to the bisphosphonate, the systemic doses to be administered to control the periimplant bone density remained unknown.
In the present study, using an FEM approach, we investigate the effect of systemic bisphosphonate treatment following total hip arthroplasty (THA) on the evolution of the peri-implant bone density.

\section{MATERIALS AND METHODS}

\section{Model of Bone Remodeling}

The developed bone remodeling model [9] takes into account the bone inhomogeneity and bone transverse isotropic symmetry by using two field variables: the relative density $\phi$ and the anisotropy tensor $\mathbf{M}$. To relate the bone adaptation to the mechanical stress environment, the temporal evolution of the relative bone density $\dot{\phi}=\mathrm{d} \phi / \mathrm{d} t$ is linked to the mechanical stimulus $\psi$ applied to the bone by a piecewise linear evolution relation (Fig. 1). The anisotropy tensor $\mathbf{M}$ is kept constant with time. This hypothesis is justified by the fact that the time period over which our model is running is short as compared to the rate of variation of the anisotropy in human bone [10]. In a period of 10 years, the anisotropy changes about by $10 \%$ while our model is simulating duration of about 1.5 years [11].

*Corresponding author. Tel.: +41-21-693-8341. Fax: +41-21-693-8660. E-mail: dominique.pioletti@epfl.ch 


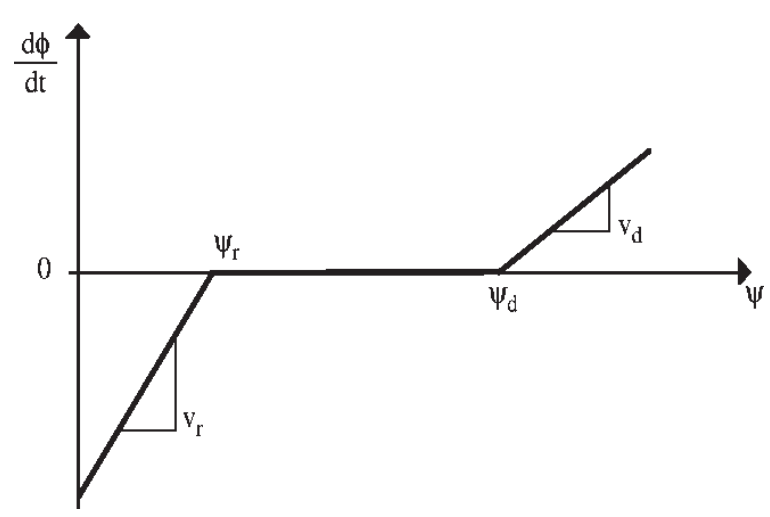

FIGURE 1 Bone relative density evolution in function of mechanical stimulus.

Moreover, numerically, changes in time of the anisotropy tensor did not significantly change the results, but the constant tensor shortens the calculation time [12]. In long bones like the femur, the anisotropy tensor can be considered as spatially constant.

An equilibrium zone, where bone neither resorbs nor densifies is delimited by two threshold stimuli $\psi_{\mathrm{r}}$ and $\psi_{\mathrm{d}}$. $v_{\mathrm{r}}$ and $v_{\mathrm{d}}$ are the slopes of the resorption and densification rates respectively versus $\psi$. The bone density adaptation function is then determined by the four parameters which are $v_{\mathrm{r}}, \psi_{\mathrm{r}}, v_{\mathrm{d}}$ and $\psi_{\mathrm{d}}$ (Fig. 1). The stimulus $\psi$ was set to a plastic yield stress [9], which is a way of measuring the microdamage, since plastic deformations are needed to create microcracks [12].

The equation describing the bone adaptation behaviour in our model is [10]:

$$
\dot{\phi}= \begin{cases}v_{\mathrm{r}}\left(\psi-\psi_{\mathrm{r}}\right) & \psi<\psi_{\mathrm{r}} \\ 0 & \psi_{\mathrm{r}} \leq \psi \leq \psi_{\mathrm{d}} \\ v_{\mathrm{d}}\left(\psi-\psi_{\mathrm{d}}\right) & \psi>\psi_{\mathrm{d}}\end{cases}
$$

\section{Model of the Drug Effect}

Drugs used to control the disease of bone metabolism, e.g. bisphosphonate, affect the bone turnover [5]. We propose to model the effects of bisphosphonate by affecting the values of the resorption parameters $\Psi_{\mathrm{r}}$ and $v_{\mathrm{r}}$. The formation parameters $\Psi_{\mathrm{d}}$ and $v_{\mathrm{d}}$ are kept constant since clinically and numerically, in a situation of peri-implant osteolysis, they have only very small effects on the temporal evolution of bone density or on bone properties. In this description, we assume that bisphosphonate has no effect on the bone formation parameters. This point has been confirmed for alendronate [13].

\section{Application to Hip Arthroplasty}

\section{Geometry and FEM}

The three-dimensional geometry of a proximal femur was reconstructed from $\mathrm{CT}$ scan slices obtained from a routine clinical examination of a 70-year-old female patient. Use of the data was approved by the patient. The implant was numerically inserted under supervision of an orthopedic surgeon. The initial bone density distribution corresponds to the density distribution obtained by the CT-scan and can be considered as the clinical situation immediately after implant insertion. Then, a finite element model of the bone-implant system was obtained with a 3D mesh generator [14] from the CT scan slices.

The FE mesh was based on 8-node isoparametric elements and consisted of 21,854 nodes and 8028 elements. At the distal end of the femur, the displacements of the nodes were constrained. Each iteration took about 30 min of calculation on a Silicon Graphics Origin 2000, using 1 out of 8 processors.

A discontinuous frictional contact between bone and implant was included, allowing us to evaluate shear micromotion between them. The used friction law was an implementation of the Coulomb's law. The friction coefficient was 0.2 . The implant's surface was considered as master surface and the bone's surface as slave surface.

Every iteration corresponds to a different time step, since the "step doubling" technique is used [9]. The evolution equation was iteratively solved by custom-made software REM [12] driving ABAQUS (Hibbitt, Karlsson, \& Sorensen Inc., Newpark, USA) analysis program.

The forces used to simulate muscle action on the head of the implant have been experimentally determined [15-17]:

Gluteus Maximus: 1901N

Gluteus Medius: $1237 \mathrm{~N}$

Psoas: $\quad 771 \mathrm{~N}$

\section{Drug Application}

In the present study, four concentrations of alendronate treatment following THA were simulated: normal bone remodeling ( $\Psi_{\mathrm{r}}$ and $v_{\mathrm{r}}$ corresponding to placebo identified values) and bone remodeling with 5, 10, $20 \mathrm{mg}$ systemic alendronate treatment ( $\Psi_{\mathrm{r}}$ and $v_{\mathrm{r}}$ corresponding to 5,10 , $20 \mathrm{mg}$ alendronate identified values).

The numerical values used for the normal bone remodeling (corresponding to the placebo case) in this study have been experimentally determined [12,18,19]. In these experiments, the authors measured the bone mineral density in patients who had one limb immobilized during convalence of a fracture. The bone mineral density was also measured once the limb was bearing weight. This results were then plotted versus time and loading history. Therefore the numerical values identified are:

$$
\begin{aligned}
& \nu_{\mathrm{r}}^{\text {placebo }}=2.800 \text { week }^{-1} \text { and } \psi_{\mathrm{r}}^{\text {placebo }}=7.5 \times 10^{-3} \\
& \nu_{\mathrm{r}}^{\text {placebo }}=0.805 \text { week }^{-1} \text { and } \psi_{\mathrm{d}}^{\text {placebo }}=3.0 \times 10^{-2}
\end{aligned}
$$


TABLE I Ratio of remodeling parameters used to simulate effect of oral alendronate treatment on bone remodeling

\begin{tabular}{lcc}
\hline Alendronate dose & $\frac{\Psi_{\mathrm{r}}^{\mathrm{r}}}{\Psi_{\mathrm{r}}^{\text {llaccoo }}} \mathrm{mg}$ & $\frac{\nu_{\mathrm{r}}^{\times}}{\nu_{\mathrm{r}}^{\mathrm{racccbo}}} \mathrm{mg}$ \\
\hline Placebo & 1.00 & 1.00 \\
$5 \mathrm{mg}$ & 0.81 & 1.07 \\
$10 \mathrm{mg}$ & 0.75 & 1.17 \\
$20 \mathrm{mg}$ & 0.72 & 1.19 \\
\hline
\end{tabular}

The numerical values for the bone remodeling following alendronate treatment were obtained from a theoretical work [20] based on a phase III clinical trial of alendronate [6]. The parameters used in order to simulate the effect of orally administrated alendronate are given in Table I [20].

\section{Results Visualization}

In order to visualize the results, we define the mean relative density (MRD) as the sum of the densities at all the nodes of the considered part divided by the number of nodes present in the considered part.

In order to visualize the effect of drug concentration on the micromovement at the bone-implant interface, we define the average interface micromovement (AIM) as the sum of the norms of the interfaces micromovements (movements between implant surface and inside bone surface) at the bone-implant interface at all the nodes of the interface divided by the number of nodes present in the considered part. AIM is expressed in $\mu \mathrm{m}$.

The MRD and AIM were reported according to the grouped Gruen zones, defined as shown in Fig. 2.

\section{RESULTS}

The use of the parameters modeling the effect of alendronate on bone remodeling increased the MRD by $2.5 \%$ for the $5 \mathrm{mg}$ dose, $3.5 \%$ for the $10 \mathrm{mg}$ dose and $4.0 \%$ for the $20 \mathrm{mg}$ dose as compared to the placebo case after

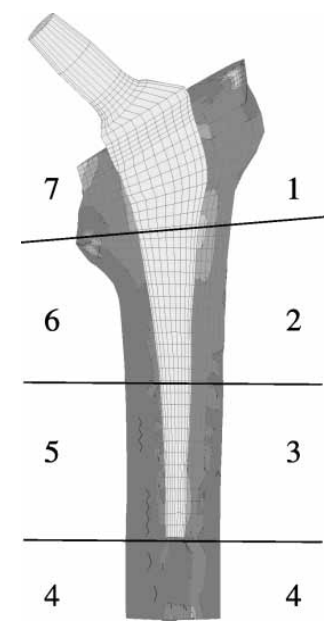

FIGURE 2 Gruen zones.
60 weeks when considering all Gruen zones (Fig. 3). At all time, the peri-implant MRD is higher in the models that were simulating alendronate treatment.

The simulated systemic alendronate treatment increases the bone density in the whole femur in a dose dependent manner when compared to the placebo case (Fig. 4). The proximal femur (Gruen zones 1 and 7; see Table II), where bone resorption takes place, experienced a smaller resorption when alendronate presence is simulated than in the placebo. The zones 2-6 also experience a higher MRD but in a lesser extent. In the distal femur, where densification takes place, the densification is only slightly increased. The increase is dose dependent but the main increase in MRD is between placebo and the $5 \mathrm{mg}$ case.

The AIM at the bone implant interface is strongly reduced in the cases where alendronate treated bone is simulated. At equilibrium, the reduction is $62 \%$ and only slightly dose dependent (Table III).

The strongest AIM decrease takes place in the proximal femur, namely the Gruen zones 1, 2, 6 and 7 (Table IV). The AIM decreases in the zones 3 and 5 is $10 \%$ lower than the decreases in the zones 1, 2, 6 and 7. The AIM decrease is only slightly dose dependent. The AIM in the Gruen zone 4 is zero because no interface is present in this zone.

\section{DISCUSSION}

There is a need to increase the lifespan of hip implants especially for younger patients. For this purpose, Shanbhag et al. [7] suggested the use of systemic bisphosphonate treatment to inhibit wear debris osteolysis. A canine THA model showed promising results. However, the use of bisphosphonate bears the danger of side effects (throat damage, ulcer) and undesired systemic skeletal effects on bone remodeling.

Therefore, a minimal bisphosphonate dose must be used. This dose will be a trade off between a sufficient increase of the implant stability through the control of bone remodeling control and minimal unwanted side effects.

To evaluate the minimal dose, we used an existing model developed by our group for calculation of bone density around an implant during remodeling [9,21] combined with bone remodeling parameters based on the identification of data from clinical studies [20]. This tool was used to simulate the effect of oral bisphosphonate treatment on the peri-implant bone density following THA.

Our model showed that all three alendronate doses decreased the peri-implant bone resorption but did not suppress it, which is confirmed by clinical results obtained by Wilkinson [8] in a clinical trial with pamidronate. The trends and the shapes of the curves representing the temporal evolution of the bone mineral density obtained in the clinical study and with our model are the same. By looking closer to the different zones and by adjusting 


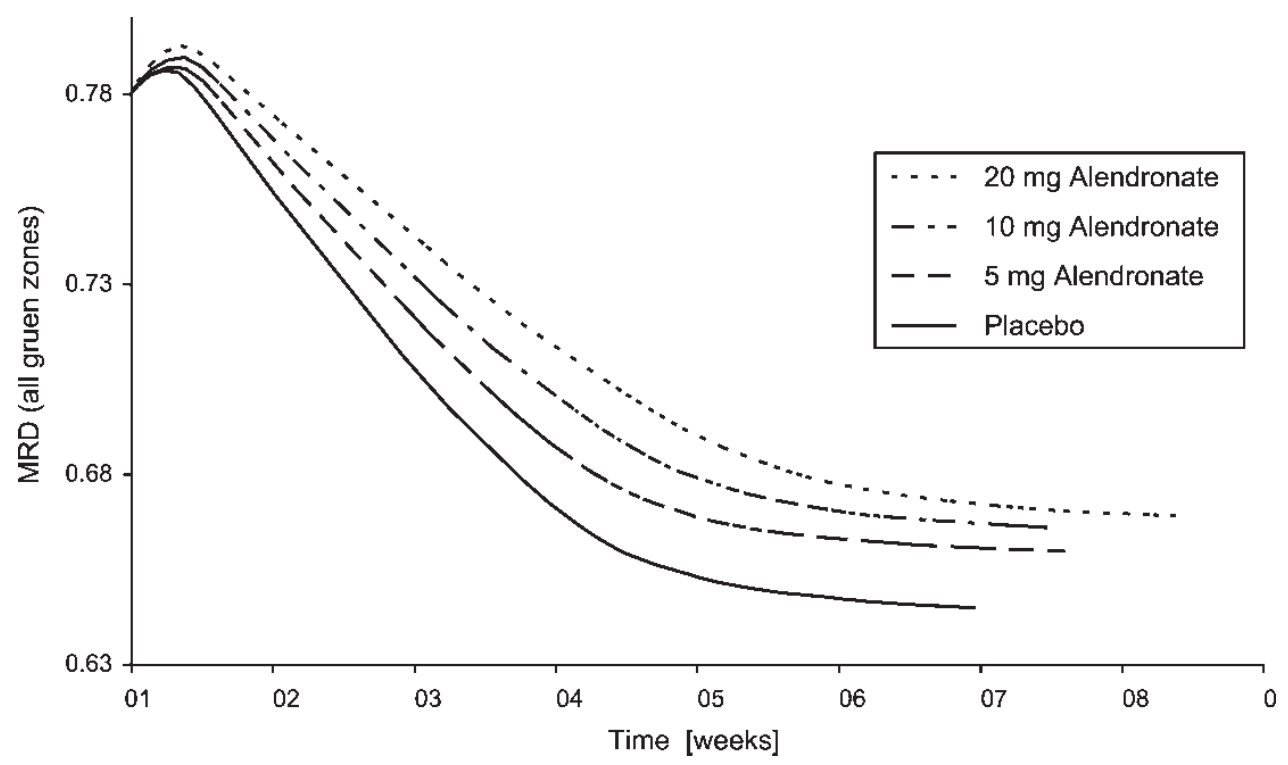

FIGURE 3 Evolution of the mean density alendronate doses until equilibrium is reached (condition which stops the simulation).

the absolute values to relative values, we noticed that the effect of the bisphosphonate measured in the clinical study [8] and in our numerical study are of the same order of magnitude. The differences between both studies can be explained by different implant geometries, different initial bone density distribution and differences between our patient and the patients in the clinical study. The results also showed that alendronate doses above $10 \mathrm{mg}$ are only a little more effective than doses below $10 \mathrm{mg}$, which is confirmed by Libermann [6].

In this study, it has been assumed that alendronate treatments had no effect on the bone formation parameters. Experimentally, it has been observed that beside resorption, formation may also decrease when bisphosphonates was used [22]. Nevertheless, no evidence for reduced osteoblastic activity at individual bone formation sites was found. Specifically, effect of alendronate on bone formation has not been observed [13]. No adverse effects on bone structure or mineralization was observed, alendronate preserved the biomechanical properties of the bone.

Moreover, the variation of bone formation parameters (clinically and numerically) has only a very small influence on the bone remodeling around an implant since most of the peri-implant bone is in resorption.

Since the equilibrium peri-implant bone distribution is dependent on parameters like initial bone density or type of implant, our model can be used to optimize the dosage for a systemic alendronate treatment. Taking into account these parameters, the goal would be to
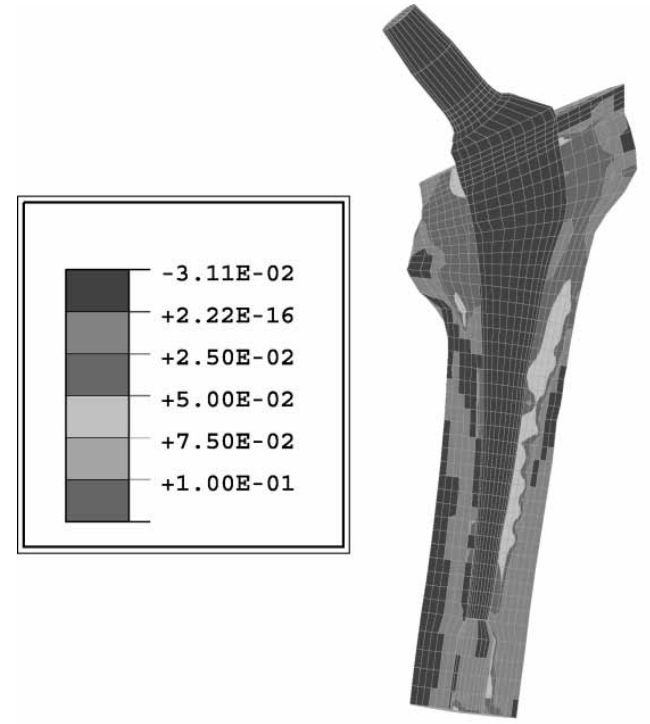

$5 \mathrm{mg} /$ Placebo

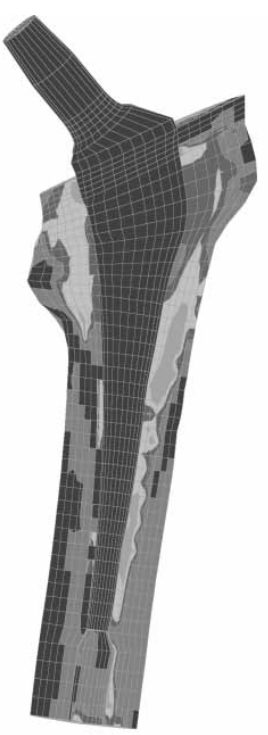

$10 \mathrm{mg} /$ Placebo

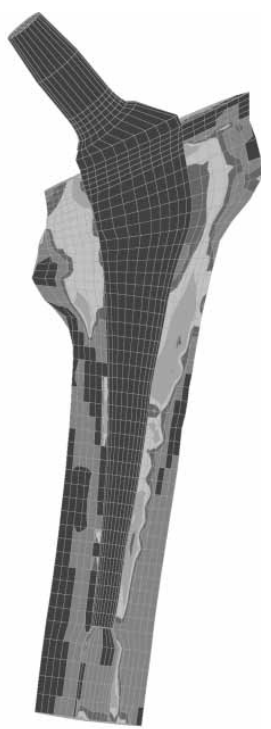

$20 \mathrm{mg} /$ Placebo

FIGURE 4 Node by node difference between the relative densities in the placebo case and the three different alendronate doses cases. 
TABLE II MRD increase relative to placebo for each Gruen zone

\begin{tabular}{lcrr}
\hline Gruen zone $(\%)$ & $5 \mathrm{mg}$ & $10 \mathrm{mg}$ & $20 \mathrm{mg}$ \\
\hline $1 / 7$ & 7.0 & 10.4 & 12.0 \\
$2 / 6$ & 3.2 & 4.3 & 4.9 \\
$3 / 5$ & 1.0 & 1.4 & 1.6 \\
4 & 0.4 & 0.5 & 0.5 \\
\hline
\end{tabular}

TABLE III AIM for the four simulated cases

\begin{tabular}{lcccc}
\hline Alendronate dose $(\mu \mathrm{m})$ & Placebo & $5 \mathrm{mg}$ & $10 \mathrm{mg}$ & $20 \mathrm{mg}$ \\
\hline AIM & 110.0 & 41.3 & 41.1 & 41.0 \\
\hline
\end{tabular}

TABLE IV Difference between AIM in placebo case and alendronate cases in each Gruen zone

\begin{tabular}{lrrrr}
\hline Gruen zone $(\mu \mathrm{m})$ & $0 \mathrm{mg}$ & $5 \mathrm{mg}$ & $10 \mathrm{mg}$ & $20 \mathrm{mg}$ \\
\hline $1 / 7$ & 111.6 & 39.4 & 39.2 & 39.0 \\
$2 / 6$ & 108.3 & 38.0 & 37.8 & 37.7 \\
$3 / 5$ & 110.2 & 46.3 & 46.1 & 46.1 \\
4 & 0.0 & 0.0 & 0.0 & 0.0 \\
\hline
\end{tabular}

obtain the equilibrium density which gives the longest possible service life of the implant with a minimal risk for the patient due to side effects of alendronate.

One of the reasons for the failure of hip arthroplasty is osteolysis due to wear particles. A source of the wear particles is the relative movement of the implant to the surrounding bone. Moreover, micromovements between the implant and the adjacent bone will lead to the encapsulation of the implant by fibrous tissue. Therefore, a very interesting point shown by this study is that interfacial micromovements are diminished due to bisphosphonate systemic treatment. The bisphosphonate dose does not influence the micromovements like it does influence the average bone density. This is probably due to the fact that the micromovements are only influenced by the bone very close to the implant surface, while the average bone density is influenced by the whole bone. Systemic bisphosphonate treatment would allow the increase of the lifespan of the implant because the alendronate treatment would allow to partially inhibit the peri-implant bone resorption and the micromovements at the bone-implant interface.

\section{CONCLUSION}

This study shows that the modification of the remodeling behavior of bone in patients undergoing a bisphosphonate treatment may allow to increase the lifespan of a hip implant by decreasing the bone resorption and by decreasing the micromovements at the bone-implant interface. Therefore the evolution towards fibrous tissues at the bone-implant interface would also be adverted [23]. Incorporation of drug effect in numerical studies of bone remodeling is a promising tool especially to predetermine safe bisphosphonate doses used in orthopedic implants in order to increase the lifespan of the implant and the quality of life of the patient.

\section{References}

[1] Amstutz, H., Dorey, F.J. and Finerman, G.A.M. (1998) In: Finerman, G.A.M., Dorey, F.J., Grigoris, P. and McKellop, H.A., eds, Total Hip Arthroplasty Outcomes (Churchill Livingston, New York), pp 55-63.

[2] Huiskes, R. (1987) "Finite element analysis of acetabular reconstruction. Noncemented threaded cups", Acta Orthop. Scand. 58, 620-625.

[3] Friedman, R.J., Black, J., Galante, J.O., Jacobs, J.J. and Skinner, H.B. (1994) "Current concepts in orthopaedic biomaterials and implant fixation", Instr. Course Lect. 43, 233-255.

[4] Pioletti, D.P., Leoni, L., Genini, D., Takei, H., Du, P. and Corbeil, J. (2002) "Gene expression analysis of osteoblastic cells contacted by orthopedic implant particles", J. Biomed. Mater Res. 61, 408-420.

[5] Fleisch, H. (1995) Bisphosphonates in Bone Disease, From The Laboratory to The Patient (The Parthenon Publishing Group, New York).

[6] Liberman, U.A., Weiss, S.R., Broll, J., Minne, H.W., Quan, H., Bell, N.H., Rodriguez-Portales, J., Downs, Jr., R.W., Dequeker, J. and Favus, M. (1995) "Effect of oral alendronate on bone mineral density and the incidence of fractures in postmenopausal osteoporosis. The Alendronate Phase III Osteoporosis Treatment Study Group", N. Engl. J. Med. 333, 1437-1443.

[7] Shanbhag, A.S., Hasselman, C.T., Kovach, C.J. and Rubash, H.E. (1997) "The John Charnley Award. Inhibition of wear debris mediated osteolysis in a canine total hip arthroplasty model", Clin. Orthop., 33-43.

[8] Wilkinson, J.M., Stockley, I., Peel, N.F., Hamer, A.J., Elson, R.A., Barrington, N.A. and Eastell, R. (2001) "Effect of pamidronate in preventing local bone loss after total hip arthroplasty: arandomized, double-blind, controlled trial", J. Bone Miner. Res. 16, 556-564.

[9] Terrier, A., Rakotomanana, R.L., Ramaniraka, R.N. and Leyvraz, P.F. (1997) "Adaptation models of anisotropic bone", Comp. Meth. Biomech. Biomed. Eng. 1, 47-59.

[10] Ding, M. and Hvid, I. (2000) "Quantification of age-related changes in the structure model type and trabecular thickness of human tibial cancellous bone", Bone 26(3), 291-295.

[11] Ding, M., Odgaard, A., Linde, F. and Hvid, I. (2002) "Age-related variations in the microstructure of human tibial cancellous bone", J. Orthop. Res. 20, 615-621.

[12] Terrier, A., PhD Thesis 2048, Swiss Federal Institute of Technology (Lausanne).

[13] Chavassieux, P.M., Arlot, M.E., Reda, C., Wei, L., Yates, A.J. and Meunier, P.J. (1997) "Histomorphometric assessment of the longterm effects of alendronate on bone quality and remodeling in patients with osteoporosis", J. Clin. Investig. 100, 1475-1480.

[14] Rubin, P.J., Leyvraz, P.F., Aubaniac, J.M., Argenson, J.N., Esteve, P. and de Roguin, B. (1992) "The morphology of the proximal femur. A three-dimensional radiographic analysis", J. Bone Jt. Surg. [Br] $\mathbf{7 4}, 28-32$

[15] Crowninshield, R.D. and Brand, R.A. (1981) "A physiologically based criterion of muscle force prediction in locomotion", J. Biomech. 14, 793-801.

[16] Davy, D.T., Kotzar, G.M., Brown, R.H., Heiple, K.G., Goldberg, V.M., Heiple, Jr., K.G., Berilla, J. and Burstein, A.H. (1988) "Telemetric force measurements across the hip after total arthroplasty", J. Bone Jt. Surg. [Am] 70, 45-50.

[17] Goldberg, V.M., Davy, D.T., Lotzar, G.L., Heiple, K.G., Brown, R.H., Berilla, J. and Burstein, A.H. (1988) In: Fitzgerald, R., eds, In Vivo Hip Forces. Non-cemented Total Hip Arthroplasty (Raven Press, New York), pp 251-256. 
[18] Nauenberg, T., Bouxsein, M.L., Mikic, B. and Carter, D.R. (1993) Proceeding of the 39th Meeting Orthopaedic Research Society San-Francisco.

[19] Rubin, C.T. and Lanyon, L.E. (1985) "Regulation of bone mass by mechanical strain magnitude", Calcif. Tissue Int. 37, 411-417.

[20] Pioletti, D.P. and Rakotomanana, L.R. (2004) "Can the increase of bone mineral density following bisphosphonates treatments be explained by biomechanical considerations?", J. Clin. Biomech. 19 $170-174$.
[21] Peter, B., Pioletti, D.P., Terrier, A. and Rakotomanana, L.R. (2001) "Orthopedic implant as drug delivery system: a numerical approach", Comp. Meth. Biomech. Biomed. Eng. 4, 505-513.

[22] Rodan, G.A. and Fleisch, H.A. (1996) "Bisphosphonates: mechanisms of action", J. Clin. Investig. 97(12), 2692-2696.

[23] Büchler, P., Pioletti, D.P. and Rakotomanana, L.R. (2003) "Biphasic constitutive laws for biological interface evolution", Biomech Model Mechanobiol. 1, 239-249. 